\title{
The Regulation of Lipolysis in Adipose Tissue
}

\author{
Julie Serr, Xiang Li and Kichoon Lee* \\ Department of Animal Sciences, The Ohio State University, Columbus, OH 43210 USA
}

\begin{abstract}
Knowledge regarding lipid catabolism has been of great interest in the field of animal sciences. In the livestock industry, excess fat accretion in meat is costly to the producer and undesirable to the consumer. However, intramuscular fat (marbling) is desirable to enhance carcass and product quality. The manipulation of lipid content to meet the goals of animal production requires an understanding of the detailed mechanisms of lipid catabolism to help meticulously design nutritional, pharmacological, and physiological approaches to regulate fat accretion. The concept of a basic system of lipases and their co-regulators has been identified. The major lipases cleave triacylglycerol (TAG) stored in lipid droplets in a sequential manner. In adipose tissue, adipose triglyceride lipase (ATGL) performs the first and rate-limiting step of TAG breakdown through hydrolysis at the sn-1 position of TAG to release a non-esterified fatty acid (NEFA) and diacylglycerol (DAG). Subsequently, cleavage of DAG occurs via the rate-limiting enzyme hormone-sensitive lipase (HSL) for DAG catabolism, which is followed by monoglyceride lipase (MGL) for monoacylglycerol (MAG) hydrolysis. Recent identification of the co-activator (Comparative Gene Identification-58) and inhibitor $[G(0) / G(1)$ Switch Gene 2] of ATGL have helped elucidate this important initial step of TAG breakdown, while also generating more questions. Additionally, the roles of these lipolysis-related enzymes in muscle, liver and skin tissue have also been found to be of great importance for the investigation of systemic lipolytic regulation.
\end{abstract}

(Key words : Lipolysis, ATGL, HSL, MGL, CGI-58, G0S2)

\section{SIGNIFICANCE OF LIPID METABOLISM IN DOMESTIC ANIMALS}

The regulation of lipid content in food animals is essential for quality and production efficiency. In poultry and livestock, lipid metabolism is closely associated with many of the critical goals of producers and consumers, such as reduction of back fat, enhancement of lean meat, control of the proper extent of marbling, and the energy balance needed for milk/egg production. Therefore, understanding of the orchestrated mechanisms of lipid mobilization in food animal species is of extensive interest. Accordingly, studies in this area are important because they provide potential targets of genes and applicable approaches to improve animal production. Furthermore, by sharing a high conservation with their human orthologues, knowledge regarding these genes and proteins related to lipid metabolism in the livestock species could also provide insight for the development of human obesity treatments.

\section{Growth and Lean Meat Production}

Excess fat accretion in meat is costly to the producer through decreased feed efficiency since it results in nutrients being converted to undesirable fat deposits and because there is a growing affinity for leaner meat by consumers. Reducing lipid deposition in certain animal body locations has been a crucial goal of the food animal industry to increase both productivity and profit while meeting market demand.

Growth, which is determined based on direct measurement of weight gain, has a critical impact on the commercial value of livestock. The quality of food animals is mainly evaluated based on two factors: 1) the amount of muscle on the carcass, and 2) the quantity and quality of its adipose tissue. However, it is well known that domestic animals of fatty breeds have a tendency to grow slower than leaner breeds. Therefore, enhancing growth, especially lean growth, is among the essential goals of genetic improvement programs in commercial lines of animals (Hamill et al., 2012). Additionally, nutritional, pharmacological, and physiological approaches toward the reduction of undesirable fat tissue in animals are being intensively investigated. The effort to

The first 2 authors contributed equally to this work.

* Corresponding author: Dr. Kichoon Lee, Department of Animal Sciences, The Ohio State University, Columbus, OH 43210 USA. Tel: 614-688-7963, Fax: 614-292-2929, E-mail: lee.2626@osu.edu 
illustrate the mechanism of the lipolytic machinery will play an important role in reducing back fat, enhancing lean meat, and improving the overall growth rate in domestic animals.

\section{Marbling in Meat Products}

Intensive genetic selection toward lean meat production has been shown to have negative consequences on measures of palatability (tenderness, juiciness, flavor, overall acceptability) in food animals (Hamill et al., 2012). Marbling, or intramuscular lipid content, is the core factor of palatability of meat (Hovenier et al., 1993; Hausman et al., 2009). Consumer perception of texture, taste and juiciness in meat is positively correlated with intramuscular fat levels. For example, a level of $1.5 \%$ intramuscular fat content has been proposed as a threshold for pork, below which the palatability is not acceptable (Fortin et al., 2005); however, commercially important cuts of pork commonly do not meet this standard (Gjerlaug-Enger et al., 2010).

As tenderness and intramuscular fat (IMF) content are heritable traits in cattle and pigs, it is important to identify genes encoding enzymes related to the regulation of intramuscular fat (Gjerlaug-Enger et al., 2010; Jeong et al., 2012). An understanding of how IMF is controlled would provide informative suggestions for genetic selection as well as set directions to improve meat quality through animal husbandry modifications in diet, hormone treatment or lifestyle since certain nutritional, developmental, or hormonal regulations would impact lipid metabolism and ultimately benefit the ideal extent of marbling in meat products.

\section{Adipose Tissue in Animals}

Animals have evolved to store excess energy efficiently in the form of lipids to maintain energy homeostasis in times of fasting or increased energy demand. The main energy store in the body is adipose tissue, which contains over $95 \%$ of the body's total lipids (Coppack et al., 1994). Adipose tissue is a multi-depot organ that is distributed over much of the body, including subcutaneous anterior and posterior depots, and multiple visceral depots (Cinti, 2006). There are two cell types comprising these depots, brown adipose tissue (BAT) and white adipose tissue(WAT). Both brown and white adipocytes contain lipid droplets; however, they have functional and morphological dissimilarities. Specifically, brown adipose tissue cells have multiple lipid droplets, and are stimulated by noradrenaline to produce heat through energy uncoupling by uncoupling protein 1, which is uniquely expressed in BAT (Cinti, 2006; Giordano et al., 2008; Enerbäck, 2010). WAT was once thought to be an inert lipid store, but was recently identified as a dynamic organ with three important functions: (1) triglyceride storage, (2) release of free fatty acids, and (3) secretion of adipokines (Deiuliis, 2007). The respective and overall amounts of these tissues vary based on gender, age, nutritional status, and other physiological conditions (Cinti, 2006).

The function of adipose tissue in the maintenance of energy homeostasis has been well-established. More recently, adipokines have been identified, beginning with leptin in 1994 (Zhang et al., 1994). Adipokines are proteins secreted in adipose tissue that have an array of endocrine and paracrine functions including maintenance of body weight homeostasis, hunger, inflammatory response, coagulation, fibrinolysis, insulin resistance, diabetes, and atherosclerosis (Lau et al., 2005). The dysregulation of adipokines is known to be associated with the pathogenesis of obesity and is thought to be causative or contributory (Waki and Tontonoz, 2007).

Adipose tissue is primarily composed of two parts or fractions: the stromal-vascular (SV) and the fat cell fraction (FC). The SV fraction is not well-defined, but contains a large portion of preadipocytes that may differentiate to mature fat cells under appropriate conditions (Ailhaud and Hauner, 2004). The FC fraction contains mature adipocytes which, due to large lipid droplets, account for the majority of adipose tissue by volume. Indeed, adipose tissue is a more dynamic organ than once thought. The largest portion of the adipose, the adipocytes, is the component responsible for the oldest known function of adipose tissue, which is to maintain energy homeostasis.

\section{BASIC PROCESS OF LIPOLYSIS IN ADIPOSE TISSUE}

Adipose tissue lipolysis is a primary factor in the maintenance of energy homeostasis in animals. In a fed state, excess dietary non-esterified fatty acids (NEFA) or fatty acids synthesized in the liver by de novo lipogenesis are esterified to form triacylglycerols (TAG), which are then stored in the lipid droplets (LD) of mature adipocytes. Upon stimulation in response to energy demand or other needs, TAGs are hydrolyzed by lipolytic enzymes to release one 
glycerol and three NEFAs, which can subsequently be used as energy substrates, essential precursors for lipid and membrane synthesis, or cell signaling mediators. The process of releasing NEFAs from stored TAGs is called lipolysis.

Lipolysis can be stimulated by signals induced by epinephrine, norepinephrine, glucagon, growth hormone, testosterone, cortisol, and ACTH according to different nutritional/hormonal statuses or needs of the animal body. Once triggered by the lipolytic signal, a major stimulation pathway is through the 7TM receptors of the cell to activate adenylate cyclase, which increases cAMP level and further activates protein kinase $\mathrm{A}$ to stimulate lipases. The process of lipolysis can be found in all tissue and cell types; however, it is most abundant in the adipose tissue. A basic lipolytic machinery has been established through several recent discoveries and previous findings (Lass et al., 2011). A series of lipases, their co-activators and inhibitors, and a number of lipid droplet-associating proteins are regarded as major components of the lipolytic machinery, and they function by connecting to signaling pathways that mediate the process of lipolysis.

Cleavage of individual fatty acids from TAGs occurs sequentially. The first cleavage yields a diacylglycerol (DAG) and the second creates a monoacylglycerol (MAG). The first cleavage is rate-limiting, and is selectively performed by adipose triglyceride lipase (ATGL). Hormone sensitive lipase (HSL) is multi-functional and may contribute to cleavage of TAGs, though it is rate-limiting for the catabolism of DAGs. Finally, monoglyceride lipase (MGL) hydrolyzes the final fatty acid. The NEFAs are transported to other tissues including skeletal and cardiac muscles and the liver, where they undergo $\beta$-oxidation to generate ATP. Glycerol is transported to the liver to provide energy through glycolysis or gluconeogenesis.

\section{Adipose Triglyceride Lipase}

In 2004, an enzyme capable of hydrolyzing TAG was simultaneously identified by three independent groups, adipose triglyceride lipase (Zimmermann et al., 2004), desnutrin (Villena et al., 2004) or calcium-independent phospholipase A2ろ (Jenkins et al., 2004). Orthologues of ATGL have been identified in cattle, pigs, chickens, turkeys, quail, rats, humans, and mice (Zimmermann et al., 2004; Eastmond, 2006; Deiuliis et al., 2008; Lee et al., 2009; Peyot et al., 2009).
ATGL selectively cleaves the first ester bond of TAG(at sn-1 position) with 10-fold higher substrate affinity for TAG than for DAG(Zimmermann et al., 2004). ATGL also exhibits some activity as a phospholipase (Notari et al., 2006) and transacylase (Jenkins et al., 2004), but these activities are lower than its activity as a TAG hydrolase. ATGL has also been reported to act as a DAG transacylase, which generates TAG and MAG from two DAG molecules (Jenkins et al., 2004).

The expression of ATGL in multiple tissues has been evaluated in several species (Villena et al., 2004; Zimmermann et al., 2004; Lake et al., 2005; Kershaw et al., 2006; Deiuliis et al., 2008; Lee et al., 2009) and consistently reported to be greatest in adipose tissue. ATGL is expressed at lower levels in other tissues, including skeletal and cardiac muscle and testis.

ATGL is a member of the patatin-domain containing a family of proteins that was originally identified in plants (Zechner et al., 2005). The ATGL protein contains two major domains that are highly conserved across species: the patatin domain (N-terminus) and the lipid binding domain (C-terminus) (Schweiger et al., 2008 Lee et al., 2009). The $\mathrm{N}$-terminal region is predicted to be an $\alpha / \beta$-fold region and contains the active serine hydrolase motif(GXSXG) (Lass et al., 2011). The C-terminus is reportedly critical in LD binding. Truncations or mutations in the $\mathrm{C}$-terminal region of ATGL hinder co-localization with the LD; however, truncation of the C-terminus results in substantially increased TAG hydrolase activity in vitro (Schweiger et al., 2008). This information suggests that the C-terminal region of ATGL is necessary for lipid droplet binding, but may also play a role in suppressing ATGL function.

\section{Hormone Sensitive Lipase}

Hormone sensitive lipase was first identified in mammalian white adipose tissue in 1964 (Vaughan et al., 1964) and was considered to be the main lipase in adipose tissue for 40 years until ATGL was identified. Although HSL is able to act on multiple substrates (TAG, DAG, MAG, cholesterol esters and retinol esters), its highest affinity is for DAG hydrolysis (10-fold greater activity than toward TAG; Lass et al., 2011). This observation suggests that HSL may be most important in DAG hydrolysis when compared with its other substrates. Similar to ATGL, HSL is expressed in several tissues, but is highest in adipose tissues (Holm et al., 1987; 
Kraemer et al., 1993).

The HSL protein contains three domains: the N-terminus involved in protein-protein interactions and lipid binding, the catalytic domain, and a regulatory domain containing multiple phosphorylation sites (Langin et al., 1993; Osterlund 1996). The coordination of functions in these three domains is expected to modulate HSL activity (Lass et al., 2011). The C-terminus is comprised of structural $\alpha / \beta$ hydrolase folds, which are common in many lipases and esterases. Within this region is a common catalytic motif found in lipases, GXSXG (Langin et al., 1993; Osterlund et al., 1996). Site directed mutagenesis experiments have demonstrated that the presence of the serine situated in this motif is critical to HSL enzyme function(Holm et al., 1994). Within the regulatory domain, phosphorylation at up to four serine residues may enhance lipolysis and, importantly, allow HSL to translocate from the cytosol to the LD during stimulated lipolysis. In addition to being responsible for lipid binding, the N-terminus interacts directly with fatty acid binding protein 4 (FABP4), which is believed to act as an intracellular chaperone for fatty acids (Watt and Steinberg, 2008). The role of FABP4 in lipolysis is not fully understood, but its interaction with HSL has been shown to enhance lipolytic activity in vitro (Shen et al., 2001).

The LD surface is bound by a family of proteins containing perilipin, adipose differentiation-related protein and tail interacting protein domains (Watt and Steinberg, 2008). Perilipin A is one such protein that seems to be critical in regulation of HSL. During PKA stimulation, perilipin A is phosphorylated at six sites and rapidly forms a tight association with HSL (Granneman et al., 2007). Phosphorylation of perilipin A is not critical for HSL translocation to the LD; however, it does appear to be necessary for lipolytic action of HSL (Miyoshi et al., 2006). These observations imply that perilipin A may influence HSL activity through more mechanisms than simply recruitment to the LD. This may occur through LD surface remodeling for better substrate access or by increasing ATGL co-localization with the LD, thereby increasing the DAG substrate supply (Watt and Steinberg, 2008).

\section{Monoglyceride Lipase}

Monoglyceride lipase was described simultaneously with HSL (Vaughan et al., 1964) and shown to have substrate specificity for MAG, not TAG or DAG(Tornqvist and
Belfrage, 1976). Despite MGL having been identified over 40 years ago, few studies addressing its role in adipose tissue lipolysis have been published. In a study investigating the function of MGL in the endocannabinoid system, MGLdeficient mice were found to have MAG accumulation in the brain (Schlosburg et al., 2010), suggesting that MGL may function in MAG catabolism in tissues other than just the adipose. Expression of MGL mRNA is widespread among tissues and is highest in the adipose tissue, kidneys and testis (Karlsson et al., 1997). The 3D structure of MGL has recently been published (Bertrand et al, 2010; Labar et al., 2010). Additionally, MGL belongs to the $\alpha / \beta$ hydrolase fold protein superfamily, which contains the canonical lipase motif (GXSXG) (Karlsson et al., 1997). Beyond this, MGL exhibits little sequence homology with known mammalian proteins. There is also very limited information available regarding its regulation as MAG hydrolysis is not rate-limiting in the lipolytic cascade. Leptin has been shown to up-regulate mRNA expression of porcine MGL (Li et al., 2008).

\section{Regulation of Lipolysis in Muscle Tissue}

As basic components of the lipolytic machinery, the lipases ATGL and HSL and their co-activators and inhibitors are not only fundamental for lipolysis in adipose tissue, but also play important roles in the lipolytic processes in muscle tissue. In muscle tissue, these proteins follow a similar pattern of basic regulation as in adipose tissue, but exert certain unique features and functions. Components of this machinery have been identified as having a critical impact in both intramyocellular and intramuscular lipid metabolism (McGarry, 2002; DeFronzo, 2004; Zhao et al., 2009; Jeong et al., 2012).

Intramuscular fat (IMF) is a critical quality factor for meat products such as beef and pork. There has been a series of studies based on cattle and swine regarding the regulation of intramuscular TAG content. At first, proteins related to lipid accumulation were emphasized as the main determinant factors (Mourot and Kouba, 1998); however, recent studies also demonstrated the essential role of enzymes such as ATGL, HSL, and MGL in lipolysis. Thus, it has been clearly demonstrated that the composition of IMF is determined by a balance between fat accumulation and degradation in the longissimus dorsi (LM) (Bernlohr et al., 2002; Jeong et al., 2012). In addition, ATGL and HSL have been connected to inflammatory responses in the muscle, as 
they are down-regulated by tumor necrosis factor a (TNFa; Shan et al., 2009).

TAG serves as a major energy source for skeletal muscle during exercise (Smekal et al., 2003) and is also the major component of IMF that is stored within the intramuscular adipocytes among the myofibers (Pethick et al., 2004). A positive correlation was observed between IMF content and body adiposity (Jeong et al., 2012). Interestingly, it has been revealed that lipid metabolism and adipokine secretory function in swine is unique in intramuscular adipocytes when compared with subcutaneous and perirenal adipocytes under different developmental and hormonal regulations (Gardan et al., 2006).

In cattle, intramuscular fat content significantly increases in the longissimus dors imuscle between 12 and 27 months of age (Lee et al., 2007). A series of potential genetic markers were examined with respect to fatty acid availability, transport, esterification, TAG breakdown, $\beta$-oxidation, and fatty acid intracellular trafficking. Among these candidates, ATGL was strongly suggested as a genetic marker for the prediction of IMF deposition with the greatest negative correlation (Jeong et al., 2012). The lower level of ATGL expression obviously contributed to a lesser extent of lipolysis, leading to more TAG being retained as IMF. In contrast, the HSL gene showed a strong positive correlation with IMF or muscle fat content in several bovine species, indicating differential roles of ATGL and HSL in controlling lipid metabolism in skeletal muscle and adipose tissues (Jeong et al., 2012).

Furthermore, the differential expression of ATGL and HSL has been shown to be inconsistent in different breeds of swine. There have been several comparative studies focusing on the expression of lipid metabolism-related genes leading to different IMF deposition (Shan et al., 2009; Zhao et al., 2009). For instance, Wujin pigs, a typically fatty swine breed from China, demonstrate greater adipocyte diameter and IMF content than that of Landrace, which is a lean breed of swine, while also exhibiting lower mRNA and protein expression of ATGL and HSL. Additionally, the enzyme activity of HSL was found to be significantly lower in Wujin pigs (Zhao et al., 2009). Shan et al. (2009) compared a fatty swine breed from China, the Jinhua pig, with the Landrace pig. In their study, the expression of ATGL and HSL mRNA were lower in the subcutaneous adipose tissue of Jinhua pigs, but greater in the longissimus dorsi muscle when compared with the Landrace pig. These conflicting results may relate to different regulatory mechanisms among breeds of pig, but it is more likely that they expose a paradox of two theories: 1) the positive correlation in which more lipid storage leads to the up-regulation of lipolytic enzymes to catabolize stored TAG, versus 2) the negative correlation in which less lipolytic enzyme activity leads to more lipid storage. More studies are urgently needed to reveal the complete function of ATGL and HSL in muscle and adipose tissue and their overall systemic effects.

\section{REGULATION OF LIPOLYSIS THROUGH ATGL}

Being the lipase for the first and rate-limiting step of the lipolytic process, ATGL and its regulatory mechanisms have been intensively studied in recent years. As summarized in Table 1, the activity and expression of ATGL is highly regulated via transcriptional and post-translational means. Complete mechanisms for regulation of ATGL have not yet been elucidated, but the known features and functions of ATGL largely contribute to the frontier research focus of lipolysis studies.

\section{Transcriptional Regulation}

The mRNA expression of ATGL is known to be responsive to nutritional factors. Transcription of ATGL in adipose tissue is transiently increased by fasting and decreased during refeeding (Villena et al., 2004; Kershaw et

Table 1. Summary of adipose triglyceride lipase regulators (Lass et al., 2011)

\begin{tabular}{llll}
\hline mRNA down & mRNA / protein up & Activity down & Activity up \\
\hline \hline Feeding & Catecholamines & Perilipin & CGI-58 \\
Insulin & Dexamethasone & Adipophilin & Isoproteronol \\
Isoproterenol & Fasting & G0S2 & \\
mTOR1 & FoxO1 & & \\
TNFa & Thiazolidinediones & & \\
\hline
\end{tabular}


al., 2006; Serr et al., 2009). Although ATGL transcription is not affected by glucagon, insulin is known to down-regulate ATGL expression(Kim et al., 2006). In fact, insulin resistance and obesity are correlated with changes in ATGL expression (Festuccia et al., 2006; Kim et al., 2006; Jocken et al., 2007; Kershaw et al., 2007; Liu et al., 2009). ATGL has also been shown to be down-regulated by TNFa, an adipokine inflammatory marker (Kim et al., 2006, Kralisch et al., 2005), and mTor complex 1 (Chakrabarti et al., 2010). Isoproterenol, an adrenergic agonist, reduces ATGL expression, but may increase enzyme activity (Kralisch et al., 2005; Lass et al., 2011). The role of adrenergic activation of ATGL transcription is unclear, as cAMP analogues or phosphodiesterase inhibitors do not affect ATGL expression(Villena et al., 2004). Some studies have reported ATGL induction by catecholamines (Festuccia et al., 2006; Liu et al., 2009). The role of leptin in ATGL regulation is also unclear. Leptin is expected to promote lipolysis and restrain energy intake. In doing so, it up-regulates peroxisome proliferator-activated receptor-gamma (PPAR $\gamma$ ), a transcription factor activating ATGL transcription. Treatment of porcine adipocytes in vitro showed that, although mRNA expression increases with leptin, ATGL protein decreases ( $\mathrm{Li}$ et al., 2010). Transcriptional regulation of ATGL by prolonged exposure to dexamethasone has also been observed (Villena et al., 2004; $\mathrm{Xu}$ et al., 2009).

\section{Post-translation Regulation}

\section{(1) Activation of ATGL by Comparative Gene Identifi- cation-58 (CGI-58)}

Extracellular TAG lipases such as lipoprotein lipase and pancreatic lipases are known to be much more active in the presence of their respective coactivator proteins, apolipoprotein CII and colipase (Borgström and Erlanson, 1971; Kinnunen et al., 1977). CGI-58, which is also known as $a / \beta$-hydrolase domain-containing protein 5 , has been shown to increase the activity of ATGL (Lass et al., 2006). CGI-58 was initially identified as the causative gene in Chanarin-Dorfman Syndrome (CDS) in humans, a condition in which excess lipid accumulation occurs in multiple tissues (Chanarin et al., 1975; Lefèvre et al., 2001), suggesting a crucial role for CGI-58 in normal lipid metabolism.

CGI-58 is a member of the $\alpha / \beta$-hydrolase esterase/lipase subfamily of proteins, although it exhibits no intrinsic lipase activity (Lass et al., 2006). This group of proteins is characterized by $\alpha / \beta$ hydrolase folds containing the catalytic triad, GXSXG, through which they exert their activity (Ollis et al., 1992). In CGI-58, the active serine residue is replaced by asparagine (GXNXG), preventing it from any inherent lipolytic activity (Yamaguchi et al., 2004; Lass et al., 2006). Calculation of the 3D homology model of mouse CGI-58 predicts the typical folding pattern for $\alpha / \beta$ hydrolases (Gruber et al., 2010). CGI-58 is highly conserved across species, with most variation between sequences being found in the N-terminal region (Lass et al., 2011). A recent report has also identified the $\mathrm{N}$-terminal area as imperative to LD localization and ATGL activation. The N-terminus contains a highly hydrophobic tryptophan-rich area that may be responsible for LD association (Gruber et al., 2010).

Purified CGI-58 was shown to have no lipolytic effect. Compared to control cells, cells with overexpressed CGI-58 showed a 1.8-fold increase in hydrolase activity. Additionally, when exogenous CGI-58 was added to COS-7 cell extracts expressing ATGL, hydrolase activity increased by 20 -fold (Lass et. al., 2006). Recent studies have reported clear evidence of the positive correlation between ATGL and CGI-58 mRNA expressions in the adipose tissue of avian and porcine species under different developmental and hormonal status (Serr et al., 2011; Li et al., 2012).

It is believed that a lipid droplet-associating protein, perilipin A, mediates activation of ATGL by CGI-58 upon stimulation. Under basal conditions, perilipin A binds CGI-58, preventing its contact with ATGL on the surface of lipid droplets (Yamaguchi et al., 2004). The basal lipolysis activity of ATGL has been shown to be independent of CGI-58 (Miyoshi et al., 2008). Upon stimulation of lipolysis, perilipin A (Peri A) is phosphorylated by protein kinase A and binds to phosphorylated HSL for its activation. CGI-58 is then released by Peri A and bound to ATGL, co-localizing on the surface of lipid droplets to initiate the process of TAG breakdown. Recent studies also suggested the activation of ATGL and CGI-58 through phosphorylation by PKA; however, the detailed mechanism of this remains unclear (Subramanian et al., 2004; Pagnon et al., 2012).

Based on these findings, it is assumed that CGI-58 activates ATGL via protein-protein interaction. It was recently reported that the minimal domain of ATGL ranges until leucine 254, which can be activated by CGI-58 (Cornaciu et al., 2011). The N-terminal of CGI-58 was also shown to be crucial for its stimulation towards ATGL. Deletion of the first 29 amino acids was found to 
completely abolish the ability of CGI-58 to activate ATGL (Gruber et al., 2010). Additionally, the aforementioned N-terminal tryptophan-containing domain of CGI-58 is essential for its binding to the lipid droplets. Interestingly, the same study revealed that, even if a CGI-58 mutant could bind to ATGL, it would not enhance the hydrolysis activity of ATGL unless its LD binding (3-tryptophan) domain is intact. These findings indicated that, for CGI-58 isoforms, the ability to activate ATGL is not entirely determined by their ATGL binding capacity, but also by their ability to localize to the lipid droplet (Gruber et al., 2010). Accordingly, the lipid droplet co-localization feature of CGI-58 is assumed to be essential to ATGL activation.

A study of murine CGI-58 identified an alternative splicing variant of CGI-58 lacking the second and third of seven exons (Yang et al., 2010a). This deleted area includes the three tryptophan residues predicted to be necessary for LD binding (Gruber et al., 2010) and the pseudo-lipase sequence (GXNXG). The short form of murine CGI-58 is not able to localize with the LD or activate ATGL, suggesting that the missing motifs are necessary for normal activity in lipolysis (Yang et al., 2010a). In addition, a recent study reported that an alternatively spliced isoform with an exon 3 deletion was present in swine (Li et al., 2012). Interestingly, this unique isoform contains the 2nd exon with lipid droplet-binding domain, but lacks the major $\mathrm{a} / \mathrm{b}$ hydrolase structure as well as the $\mathrm{C}$-terminus due to an open reading frame (ORF) shift that creates a premature stop codon. However, a study of avian species did not identify any alternatively spliced variants of CGI-58 in the chicken, turkey, or quail (Serr et al., 2011). Accordingly, the impact of CGI-58 alternative splicing events on regulation of ATGL's lipolytic activity still needs to be further elucidated.

\section{Inhibition of ATGL by $G(0) / G(1)$ Switch Gene 2 (G0S2)}

A protein was very recently identified as a selective inhibitor of ATGL (Yang et al., 2010b). Originally, G0S2 was identified in blood mononuclear cells as its mRNA expression was observed with the re-entry of cells from G0 into the G1 phase in the cell cycle (Russell and Forsdyke, 1991). Later, its increased expression was observed during differentiation of 3T3-L1 adipocytes and of human preadipocytes, indicating that it may play roles other than in cell proliferation (Zandbergen et al., 2005).
The G0S2 gene is predominantly expressed in adipose tissue, similar to ATGL and CGI-58 (Subramanian et al., 2004; Villena et al., 2004; Zimmermann et al., 2004; Deiuliis et al., 2008; Lee et al., 2009; Oh et al., 2010; Yang et al., 2010b). Observation of G0S2 expression during differentiation of adipocyte cell lines and chicken preadipocytes has shown that G0S2 mRNA is induced two to three days after differentiation and remains high thereafter (Zandbergen et al., 2005; Oh et al., 2010; Yang et al., 2010b). Measurement of G0S2 in SV and FC fractions of adipose tissue has shown that G0S2 is associated with mature adipocytes (Oh et al., 2010). Recent studies have shown that G0S2 expression is attenuated by factors stimulating lipolysis, including $\mathrm{TNFa}$, isoproterenol, $\operatorname{PPAR} \gamma$, and fasting (Zandbergen et al., 2005; Oh et al., 2010; Yang et al., 2010b). In contrast, G0S2 expression is induced by insulin and refeeding (Oh et al., 2010; Yang et al., 2010b).

Direct interaction of G0S2 and the N-terminal patatin domain of ATGL has been reported (Yang et al., 2010b), but G0S2 does not appear to directly compete with CGI-58 binding at the N-terminus of ATGL (Schweiger et al., 2008; $\mathrm{Lu}$ et al., 2010). However, G0S2 is capable of preventing ATGL-mediated lipolysis in the presence of CGI-58, suggesting that G0S2 may be the dominant regulator $(\mathrm{Lu}$ et al., 2010). During stimulated lipolysis, G0S2 and ATGL translocate to the LD(Fig. 1), while inhibition of ATGL expression impairs G0S2 recruitment to the LD(Yang et al., 2010b). These findings suggest that G0S2 may not bind to the LD alone and that it may require ATGL as a binding partner (Lass et al., 2011). The current belief is that differential control of ATGL by CGI-58 and G0S2 is necessary for efficient regulation of lipolysis (Lu et al., 2010); however, further research is needed to elucidate the mechanisms involved.

\section{Pathogenesis of Mutations in ATGL and CGI-58}

The importance of ATGL and CGI-58 in the mobilization of TAG stores in adipose tissue has been established. However, disruption of these genes does not only result in dysregulation in WAT, but also in the entire body. Neutral lipid storage disease (NLSD) is a rare nonlysosomal, autosomal recessive lipid storage disorder characterized by systemic TAG deposition in leukocytes, muscle, liver, fibroblasts, and other tissues (Charanin et al., 1975; Schweiger et al., 2009). 


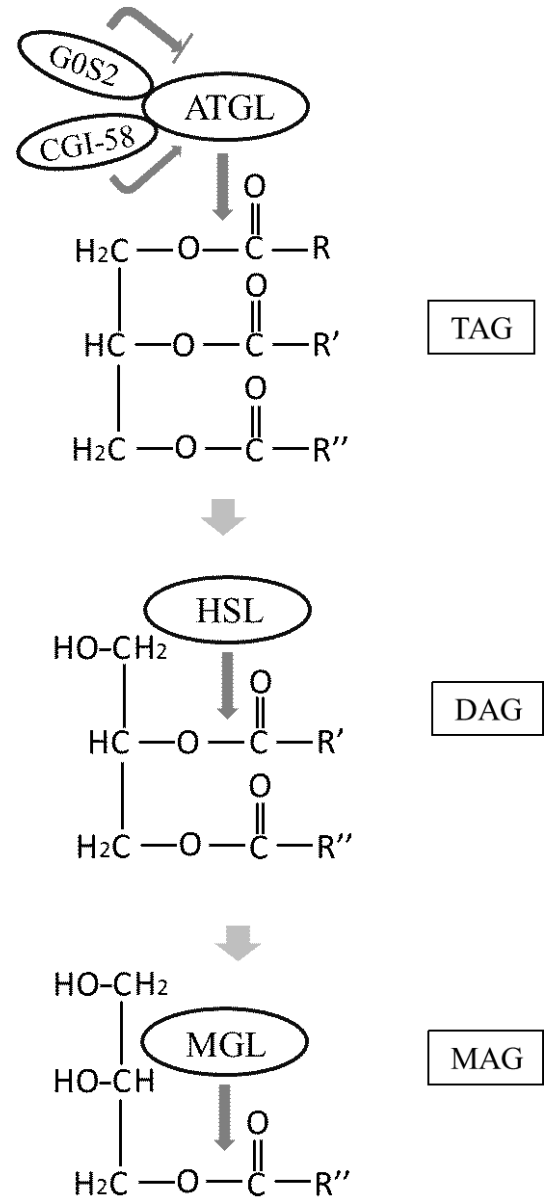

Fig. 1. Three major lipases in the process of TAG breakdown.

TAG: triacylglycerol; DAG: diacylglycerol; MAG: monoacylglycerol; ATGL: adipose triglyceride lipase; HSL: Hormone sensitive lipase; MGL: monoglyceride lipase.

Two variations of NLSD have been observed. Specifically, mutations of the CGI-58 gene have been clearly linked to NLSD with ichthyosis (NLSDI), which is also known as Chanarin-Dorfman Syndrome (CDS) (Charanin et al., 1975; Lefevre et al., 2001), while deficiency of ATGL has been shown to be correlated with NLSD with myopathy (NLSDM) (Fischer et al., 2007). Patients with NLSD caused by ATGL mutations do not exhibit ichthyosis, suggestive of a role for CGI-58 in skin barrier development in addition to TAG mobilization. Unlike ATGL, normal CGI-58 possesses acyl-CoA dependent lysophosphatidic acid acyl-transferase activity (LPAAT) (Ghosh et al., 2008; Montero-Moran et al., 2010), which is dependent on a C-terminal motif. In addition to this unique function, CGI-58 has also been shown to be associated with indispensable roles in formation of the skin permeability barrier, development of the epidermis, and even with an ATGL-independent role in epidermal TAG hydrolysis (Radner et al., 2011).

Although the impact of ATGL and CGI-58 mutations in livestock animals remains largely unexplored, the pathogenesis of ATGL and CGI-58 in humans strongly supports that further intensive studies of the lipolytic machinery components in tissues other than the adipose, such as muscle, liver, and skin, may benefit human health research as well as animal production.

\section{CONCLUSIONS}

Great progress has been made in describing the major components of the lipolytic machinery, the major lipases ATGL, HSL and MGL, and their co-activators and inhibitors such as CGI-58 and G0S2. However, further exploration of the processes of lipolysis has led to the generation of more complex questions. For instance, adipose tissue served as a representative model of the regulation of the major lipases, but the detailed interactions between lipases and their coregulators have not been fully described in adipose tissue. The lipolysis stimulation signaling pathway also remains unclear. Furthermore, the lipolytic processes in other body compartments such as the muscle, liver, or skin have been shown to be similar, yet still vary from the adipose lipid metabolism. Especially for ATGL, HSL and CGI-58, their tissue-specific regulatory mechanisms provide questions with great value to be examined, which will contribute to the overall systemic coordination of lipid metabolism under physiological, nutritional, hormonal, or developmental conditions.

An understanding of lipolytic machinery at the molecular and cellular levels is essential to developing applicable approaches to control the lipid content in animal products. Studies of related topics have provided evidence that these lipases and their co-regulators are potential targets for development of methods of controlling adiposity, which is essential for higher quality animal products, higher efficiency and increased profits.

\section{ACKNOWLEDGMENTS}

This work was supported by the Edna Jaap Poultry Endowment Fund (K Lee), the Agriculture and Food Research Initiative Competitive Grant (No. 2010-65206-20716) from the USDA National Institute of Food and Agriculture (K Lee), 
the Ohio Agricultural Research and Development Center SEEDs research funding (K Lee), and the National Research Foundation of Korea Grant funded by the Korean government (KRF-2009-220-F00006). We are grateful to Michelle Milligan (Department of Animal Sciences, The Ohio State University) for her aid in editing this manuscript.

\section{REFERENCES}

Ailhaud, G. and Hauner, H. 2004. Development of white adipose tissue. Handbook of Obesity, Etiology and Pathophysiology, 2nd edition, eds. G. A. Bray and C. Bouchard, Marcel Dekker, New York, NY, pp. 48.

Bernlohr, D. A., Jenkins, A. E. and Bennaars, A. A. 2002. Adipose tissue and lipid metabolism. Fourth edition ed. Vance, D. E., Vance, J. E., Biochemistry of Lipids, Lipoproteins and Membrances, Elsevier, Amsterdam, pp. 263-289.

Bertrand, T., Auge, F., Houtmann, J., Rak, A., Vallee, F., Mikol, V., Berne, P. F., Michot, N., Cheuret, D., Hoornaert, C. and Mathieu, M. 2010. Structural basis for human monoglyceride lipase inhibition. J. Mol. Biol. 396:663-673.

Borgström, B. and Erlanson, C. 1971. Pancreatic juice co-lipase: physiological importance. Biochim. Biophys. Acta. 20:509-513.

Chakrabarti, P., English, T., Shi, J., Smas, C. M. and Kandror, K. V. 2010. Mammalian target of rapamycin complex 1 suppresses lipolysis, stimulates lipogenesis, and promotes fat storage. Diabetes 59:775-781.

Chanarin, I., Patel, A., Slavin, G., Willis, E. J., Andrews, T. M. and Stewart, G. 1975. Neutral-lipid storage disease: a new disorder of lipid metabolism. Br. Med. J. 1:553-555.

Cinti, S. 2006. The role of brown adipose tissue in human obesity. Nutr. Metab. Cardiovasc. Dis. 16:569-574.

Cornaciu, I., Boeszoermenyi, A., Lindermuth, H., Nagy, H. M., Cerk, I. K., Ebner, C., Salzburger, B., Gruber, A., Schweiger, M., Zechner, R., Lass, A., Zimmermann, R. and Oberer, M. 2011. The minimal domain of adipose triglyceride lipase (ATGL) ranges until leucine 254 and can be activated and inhibited by CGI-58 and G0S2, respectively. PLoS One 6:26349.

Coppack, S. W., Jensen, M. D. and Miles, J. M. 1994. In vivo regulation of lipolysis in humans. J. Lipid Res. 35:177-193.

DeFronzo, R. A. 2004. Pathogenesis of type 2 diabetes mellitus. Med. Clin. North Am. 88:787-835.

Deiuliis, J. A. 2007. The metabolic and molecular regulation of adipose triglyceride lipase. Ph. D. Dissertation. The Ohio State University, Columbus.

Deiuliis, J. A., Shin, J., Bae, D., Azain, M. J., Barb, R. and Lee,
K. 2008. Developmental, hormonal, and nutritional regulation of porcine adipose triglyceride lipase (ATGL). Lipids 43:215-225.

Eastmond, P. J. 2006. Sugar-dependent1 encodes a patatin domain triacylglycerol lipase that initiates storage oil breakdown in germinating Arabidopsis seeds. Plant Cell. 18:665675.

Enerbäck, S. 2010. Human brown adipose tissue. Cell Metab. 11:248-252.

Festuccia, W. T., Laplante, M., Berthiaume, M., Gelinas, Y. and Deshaies, Y. 2006. PPARgamma agonism increases rat adipose tissue lipolysis, expression of glyceride lipases, and the response of lipolysis to hormonal control. Diabetologia. 49: 2427-2436.

Fischer, J., Negre-Salvayre, A. and Slavayre, R. 2007. Neutral lipid storage diseases and ATGL (adipose triglyceride lipase) and CGI-58/ABHD5 (alpha-beta hydrolase domain-containing 5) deficiency: myopathy, ichthyosis, but not obesity. Med. Sci. (Paris). 23:575-578.

Fortin, A., Robertson, W. M. and Tong, A. K. W. 2005. The eating quality of Canadian pork and its relationship with intramuscular fat. Meat Sci. 69:297-305.

Gardan, D., Gondret, F. and Louveau, I. 2006. Lipid metabolism and secretory function of porcine intramuscular adipocytes compared with subcutaneous and perirenal adipocytes. Am. J. Physiol. Endocrinol. Metab. 291:372-380.

Ghosh, A. K., Ramakrishnan, G., Chandramohan, C. and Rajasekharan, R. 2008. CGI-58, the causative gene for Chanarin-Dorfman syndrome, mediates acylation of lysophosphatidic acid. J. Biol.Chem. 283:24525-24533.

Giordano, A., Frontini, A. and Cinti, S. 2008. Adipose organ nerves revealed by immunohistochemistry. Methods Mol. Biol. 456:83-95.

Gjerlaug-Enger, E., Aass, L., Odegård, J. and Vangen, O. 2010. Genetic parameters of meat quality traits in two pig breeds measured by rapid methods. Animal 4:1832-1843.

Granneman, J. G., Moore, H. P., Granneman, R. L., Greenberg, A. S., Obin, M. S. and Zhu, Z. 2007. Analysis of lipolytic protein trafficking and interactions in adipocytes. J. Biol. Chem. 282:5726-5735.

Gruber, A., Cornaciu, I., Lass, A., Schweiger, M., Poeschl, M., Eder, C., Kumari, M., Schoiswohl, G., Wolinski, H., Kohlwein, S. D., Zechner, R., Zimmermann, R. and Oberer, M. 2010. The N-terminal region of comparative gene identification-58 (CGI-58) is important for lipid droplet binding and activation of adipose triglyceride lipase. J. Biol. Chem. 285:12289-12298.

Hamill, R. M., McBryan, J., McGee, C., Mullen, A. M., 
Sweeney, T., Talbot, A., Cairns, M. T. and Davey, G. C. 2012. Functional analysis of muscle gene expression profiles associated with tenderness and intramuscular fat content in pork. Meat Sci. 92:440-450.

Hausman, G. J., Dodson, M. V., Ajuwon, K., Azain, M., Barnes, K. M., Guan, L. L., Jiang, Z., Poulos, S. P., Sainz, R. D., Smith, S., Spurlock, M., Novakofski, J., Fernyhough, M. E. and Bergen, W. G. 2009. Board-invited review: the biology and regulation of preadipocytes and adipocytes in meat animals. J. Anim. Sci. 87:1218-1246.

Holm, C., Belfrage, P. and Fredrikson, G. 1987. Immunological evidence for the presence of hormone-sensitive lipase in rat tissues other than adipose tissue. Biochem. Biophys. Res. Commun. 148:99-105.

Holm, C., Belfrage, P., Osterlund, T., Davis, R. C., Schotz, M. C. and Langin, D. 1994. Hormone-sensitive lipase: structure, function, evolution and overproduction in insect cells using the baculovirus expression system. Protein Eng. 7:537-541.

Hovenier, R., Kanis, E. and Verhoeven, J. A. 1993. Repeatability of taste panel tenderness scores and their relationships to objective pig meat quality traits. J. Anim. Sci. 71:2018-2025.

Jenkins, C. M., Mancuso, D. J., Yan, W., Sims, H. F., Gibson, B. and Gross, R. W. 2004. Identification, cloning, expression, and purification of three novel human calcium-independent phospholipase A2 family members possessing triacylglycerol lipase and acylglycerol transacylase activities. J. Biol. Chem. 279:48968-48975.

Jeong, J., Kwon, E. G., Im, S. K., Seo, K. S. and Baik, M. 2012. Expression of fat deposition and fat removal genes is associated with intramuscular fat content in longissimus dorsi muscle of Korean cattle steers. J. Anim. Sci. 90:2044-20453.

Jocken, J. W., Langin, D., Smit, E., Saris, W. H., Valle, C., Hul, G. B., Holm, C., Arner, P. and Blaak, E. E. 2007. Adipose triglyceride lipase and hormone-sensitive lipase protein expression is decreased in the obese insulin-resistant state. J. Clin. Endocrinol. Metab. 92:2292-2299.

Karlsson, M., Contreras, J. A., Hellman, U., Tornqvist, H. and Holm, C. 1997. cDNA cloning, tissue distribution, and identification of the catalytic triad of monoglyceride lipase. Evolutionary relationship to esterases, lysophospholipases, and haloperoxidases. J. Biol. Chem. 272:27218-27223.

Kershaw, E. E., Hamm, J. K., Verhagen, L. A., Peroni, O., Katic, M. and Flier, J. S. 2006. Adipose triglyceride lipase: function, regulation by insulin, and comparison with adiponutrin. Diabetes 55:148-157.

Kim, J. Y., Tillison, K., Lee, J. H., Rearick, D. A. and Smas, C. M. 2006. The adipose tissue triglyceride lipase ATGL/PNPLA2 is downregulated by insulin and TNF-alpha in 3T3-L1 adipocytes and is a target for transactivation by PPARgamma. Am. J. Physiol. Endocrinol. Metab. 291:E115-127.

Kinnunen, P. K., Jackson, R. L., Smith, L. C., Gotto, A. M. Jr. and Sparrow, J. T. 1977. Activation of lipoprotein lipase by native and synthetic fragments of human plasma apolipoprotein C-II. Proc. Natl. Acad. Sci. USA. 74:4848-4851.

Kraemer, F. B., Patel, S., Sadi, M. S. and Sztalryd, C. 1993. Detection of hormone-sensitive lipase in various tissues. I. Expression of an HSL/bacterial fusion protein and generation of anti-HSL antibodies. J. Lipid Res. 34:663-671.

Kralisch, S., Klein, J., Lossner, U., Bluher, M., Paschke, R., Stumvoll, M. and Fasshauer, M. 2005. Isoproterenol, TNFalpha, and insulin downregulate adipose triglyceride lipase in 3T3-L1 adipocytes. Mol. Cell. Endocrinol. 240:43-49.

Labar, G. C., Bauvois, F. B., Ferrer, J. L., Wouters, J. and Lambert, D. M. 2010. Crystal structure of the human monoacylglycerol lipase, a key actor in endocannabinoid signaling. Chembiochem. 11:218-227.

Lake, A. C., Sun, Y., Li, J. L., Johnson, J. W., Li, D., Revett, T., Shih, H. H., Liu, W., Paulsen, J. E. and Gimeno, R. E. 2005. Expression, regulation, and triglyceride hydrolase activity of Adiponutrin family members. J. Lipid Res. 46:2477-2487.

Langin, D., Laurell, H., Holst, L. S., Belfrage, P. and Holm, C. 1993. Gene organization and primary structure of human hormone-sensitive lipase: possible significance of a sequence homology with a lipase of Moraxella TA144, an antartic bacterium. Proc. Natl. Acad. Sci. 90:4897-4901.

Lass, A., Zimmermann, R., Oberer, M. and Zechner, R. 2011. Lipolysis- a highly regulated multi-enzyme complex mediates the catabolism of cellular fat stores. Prog. Lipid Res. 50:14-27.

Lass, A., Zimmermann, R., Haemmerle, G., Riederer, M., Schoiswohl, G., Schweiger, M., Kienesberger, P., Strauss, J. G., Gokiewicz, G. and Zechner, R. 2006. Adipose triglyceride lipase-mediated lipolysis of cellular fat stores is activated by CGI-58 and defective in Chanarin-Dorfman Syndrome. Cell Metab. 3:309-319.

Lau, D. C., Dhillon, B., Yan, H., Szmitko, P. E. and Verma, S. 2005. Adipokines: molecular links between obesity and atherosclerosis. Am. J. Physiol. Heart Circ. Physiol. 288(5): H2031-41.

Lee, S. H., Park, E. W., Cho, Y. M., Kim, S. K., Lee, J. H., Jeon, J. T., Lee, C. S., Im, S. K., Oh, S. J., Thompson, J. M. and Yoon, D. 2007. Identification of differentially expressed genes related to intramuscular fat development in the early and late fattening stages of hanwoo steers. J. Biochem. Mol. Biol. 
40:757-764.

Lee, K., Shin, J., Latshaw, J. D., Suh, Y. and Serr, J. 2009. Cloning of adipose triglyceride lipase in poultry and expression of adipose triglyceride lipase during development of adipose in chickens. Poult. Sci. 88:620-630.

Lefèvre, C., Jobard, F., Caux, F., Bouadjar, B., Karaduman, A., Heilig, R., Lakhdar, H., Wollenberg, A., Verret, J. L., Weissenbach, J., Ozgüc, M., Lathrop, M., Prud'homme, J. F. and Fischer, J. 2001. Mutations in CGI-58, the gene encoding a new protein of the esterase/lipase/thioesterase subfamily, in Chanarin-Dorfman syndrome. Am. J. Hum. Genet. 69:10021012.

Lefèvre, C., Jobard, F., Caux, F., Bouadjar, B., Karaduman, A., Heilig, R., Lakhdar, H., Wollenberg, A.., Verret, J. L., Weissenbach, J., Ozgüc, M., Lathrop, M., Prud'homme, J. F. and Fischer, J. 2001. Mutations in CGI-58, the gene encoding a new protein of the esterase/lipase/thioesterase subfamily, in Chanarin-Dorfman syndrome. Am. J. Hum. Genet. 69:10021012.

Li, X., Suh, Y., Kim, B. R., Moeller, S. J. and Lee, K. 2012. Alternative splicing and developmental and hormonal regulation of porcine comparative gene identification-58 (CGI-58) mRNA. J. Anim. Sci. 90:4346-4354.

Li, Y., Zheng, X. and Yang, G. 2008. Effects of leptin on porcine primary adipocytes lipolysis and mRNA expression of key lipolytic enzymes. Sheng Wu Gong Cheng Xue Bao. 24: 1612-1619.

Li, Y. C., Zheng, X. L., Liu, B. T. and Yang, G. S. 2010. Regulation of ATGL expression mediated by leptin in vitro in porcine adipocyte lipolysis. Mol. Cell Biochem. 333(1-2):121-8.

Liu, L. F., Purushotham, A., Wendel, A. A., Koba, K., Deiuliis, J., Lee, K. and Belury, M. A. 2009. Regulation of adipose triglyceride lipase by rosiglitazone. Diabetes Obes. Metab. 11:131-142.

Lu, X., Yang, X. and Liu, J. 2010. Differential control of ATGL-mediated lipid droplet degradation by CGI-58 and G0S2. Cell Cycle. 9:2719-2725.

McGarry, J. D. 2002. Banting lecture 2001: Dysregulation of fatty acid metabolism in the etiology of type 2 diabetes. Diabetes 51:7-18.

Miyoshi, H., Perfield, J. W., Obin, M. S. and Greenberg, A. S. 2008. Adipose triglyceride lipase regulates basal lipolysis and lipid droplet size in adipocytes. J. Cell Biochem. 105:14301436.

Miyoshi, H., Souza, S. C., Zhang, H. H., Strissel, K. J., Christoffolete, M. A., Kovsan, J., Rudich, A., Kraemer, F. B., Bianco, A. C., Obin, M. S. and Greenberg, A. S. 2006.
Perilipin promotes hormone-sensitive lipase-mediated adipocyte lipolysis via phosphorylation-dependent and -independent mechanisms. J. Biol. Chem. 281:15837-15844.

Montero-Moran, G., Caviglia, J. M., McMahon, D., Rothenberg, A., Subramanian, V., Xu, Z., Lara-Gonzalex, S., Storch, J., Carman, J. G. and Brasaemle, D. L. 2010. CGI-58/ABHD5 is a coenzyme A-dependent lysophosphatidic acid acyltransferase. J. Lipid Res. 51:709-719.

Mourot, J. and Kouba, M. 1998. Lipogenic enzyme activities in muscles of growing Large White and Meishan Pigs. Livest. Prod. Sci. 55:127-133

Notari, L, Baladron, V., Aroca-Aguilar, J. D., Balko, N., Heredia, R., Meyer, C., Notario, P. M., Saravanamuthu, S., Nueda, M. L., Sanchez-Sanchez, F., Escribano, J., Laborda, J. and Becerra, S. P. 2006. Identification of a lipase-linked cell membrane receptor for pigment epithelium-derived factor. J. Biol. Chem. 281:38022-38037.

Oh, S. A., Suh, Y., Pang, M. G. and Lee, K. 2010. Cloning of avian $\mathrm{G}(0) / \mathrm{G}(1)$ switch gene 2 genes and developmental and nutritional regulation of $\mathrm{G}(0) / \mathrm{G}(1)$ switch gene 2 in chicken adipose tissue. J. Anim. Sci. 89:367-375.

Ollis, D. L., Cheah, E., Cygler, M., Dijkstra, B., Frolow, F., Franken, S. M., Harel, M., Remington, S. J., Silman, I. and Schrag, J. 1992. The alpha/beta hydrolase fold. Protein Eng. 5:197-211.

Osterlund, T., Danielsson, B., Degerman, E., Contreras, J. A., Edgren, G., Davis, R. C., Schotz, M. C. and Holm, C. 1996. Domain-structure analysis of recombinant rat hormone-sensitive lipase. Biochem. J. 319:411-420.

Pagnon, J., Matzaris, M., Stark, R., Meex, R. C., Lance Macaulay, S., Brown, W., O’Brien, P. E., Tiganis, T. and Watt, M. J. 2012. Identification and Functional Characterization of Protein Kinase A Phosphorylation Sites in the Major Lipolytic Protein, Adipose Triglyceride Lipase. Endocrinology 153:4278-4289.

Pethick, D. W., Harper, G. S., Oddy, V. H. 2004. Growth, development and nutritional manipulation of marbling in cattle: a review. Aust. J. Exp.Agric. 44:705-715.

Peyot, M. L., Guay, C., Latour, M. G., Lamontagne, J., Lussier, R., Pineda, M., Ruderman, N. B., Haemmerle, G., Zechner, R., Joly, E., Madiraju, S. R., Poitout, V. and Prentki, M. 2009. Adipose triglyceride lipase is implicated in fuel- and non-fuelstimulated insulin secretion. J. Biol. Chem. 284:16848-16859.

Radner, F. P., Grond, S., Haemmerle, G., Lass, A. and Zechner, R. 2011. Fat in the skin: Triacylglycerol metabolism in keratinocytes and its role in the development of neutral lipid storage disease. Dermatoendocrinol. 3:77-83.

Russell, L. and Forsdyke, D. R. 1991. A human putative 
lymphocyte G0/G1 switch gene containing a CpG-rich island encodes a small basic protein with the potential to be phosphorylated. DNA Cell Biol. 10:581-591.

Schlosburg, J. E., Blankman, J. L., Long, J. Z., Nomura, D. K., Pan, B., Kinsey, S. G., Nguyen, P. T., Ramesh, D., Booker, L., Burston, J. J., Thomas, E. A., Selley, D. E., Sim-Selley, L. J., Liu, Q. S., Lichtman, A. H. and Cravatt, B. F. 2010. Chronic monoacylglycerol lipase blockade causes functional antagonism of the endocannabinoid system. Nat. Neurosci. 13: 1113-1119.

Schweiger, M., Lass, A., Zimmermann, R., Eichmann, T. O. and Zechner, R. 2009. Neutral lipid storage disease: genetic disorders caused by mutations in adipose triglyceride lipase/ PNPLA2 or CGI-58/ABHD5. Am. J. Physiol. Endocrinol. Metab. 297:E289-296.

Schweiger, M., Schoiswohl, G., Lass, A., Radner, F. P., Haemmerle, G., Malli, R., Graier, W., Cornaciu, I., Oberer, M., Salvayre, R., Fischer, J., Zechner, R. and Zimmermann, R. 2008. The C-terminal region of human adipose triglyceride lipase affects enzyme activity and lipid droplet binding. J. Biol. Chem. 283:17211-17220.

Serr, J., Suh, Y. and Lee, K. 2009. Regulation of adipose triglyceride lipase by fasting and refeeding in avian species. Poult. Sci. 88:2585-2591.

Serr, J., Suh, Y. and Lee, K. 2011. Cloning of comparative gene identification-58 gene in avian species and investigation of its developmental and nutritional regulation in chicken adipose tissue. J. Anim. Sci. 89:3490-3500.

Shan, T., Wu, T., Reng, Y. and Wang, Y. 2009. Breed difference and regulation of the porcine adipose triglyceride lipase and hormone sensitive lipase by TNFalpha. Anim. Genet. 40:863870.

Shen, W. J., Liang, Y., Hong, R., Patel, S., Natu, V., Sridhar, K., Jenkins, A., Bernlohr, D. A. and Kraemer, F. B. 2001. Characterization of the functional interaction of adipocyte lipidbinding protein with hormone-sensitive lipase. J. Biol. Chem. 376:49443-49448.

Smekal, G., von Duvillard, S. P., Pokan, R., Tschan, H., Baron, R., Hofmann, P., Wonisch, M. and Bachl, N. 2003. Effect of endurance training on muscle fat metabolism during prolonged exercise: agreements and disagreements. Nutrition 19:891-900.

Subramanian, V., Rothenberg, A., Gomez, C., Cohen, A. W., Garcia, A., Bhattacharyya, S., Shapiro, L., Dolios, G., Wang, R., Lisanti, M. P. and Brasaemle, D. L. 2004. Perilipin A mediates the reversible binding of CGI-58 to lipid droplets in 3T3-L1 adipocytes. J. Biol. Chem. 279:42062-42071.

Tornqvist, H. and Belfrage, P. 1976. Determination of protein in adipose tissue extracts. FEBS Lett. 75:259-264.
Vaughan, M., Berger, J. E. and Steinberg, D. 1964. Hormonesensitive lipase and monoglyceride lipase activities in adipose tissue. J. Biol. Chem. 239: 401409.

Villena, J. A., Roy, S., Sarkadi-Nagy, E., Kim, K. H. and Sul, H. S. 2004. Desnutrin, an adipocyte gene encoding a novel patatin domain-containing protein, is induced by fasting and glucocorticoids: ectopic expression of desnutrin increases triglyceride hydrolysis. J. Biol. Chem. 279:47066-47075.

Waki, H. and Tontonoz, P. 2007. Endocrine functions of adipose tissue. Annu. Rev. Pathol. 2:31-56.

Watt, M. J. and Steinberg, G. R. 2008. Regulation and function of triacylglycerol lipases in cellular metabolism. Biochem. J. 414:313-325.

Xu, C., Jinhan, H., Jiang, H., Zu, L., Zhai, W., Pu, S. and Xu, G. 2009. Direct effect of glucocorticoids on lipolysis in adipocytes. Mol. Endocrinol. 23:1161-1170.

Yamaguchi, T., Omatsu, N., Matsushita, S. and Osumi, T. 2004. CGI-58 interacts with perilipin and is localized to lipid droplets. Possible involvement of CGI-58 mislocalization in Chanarin-Dorfman syndrome. J. Biol. Chem. 279:30490-30497.

Yang, X., Lu, X. and Liu, J. 2010a. Identification of a novel splicing isoform of murine CGI-58. FEBS Lett. 584:903-910.

Yang, X., Lu, X., Lombès, M., Rha, G. B., Chi, Y. I., Guerin, T. M., Smart, E. J. and Liu, J. 2010b. The $G(0) / G(1)$ switch gene 2 regulates adipose lipolysis through association with adipose triglyceride lipase. Cell Metab. 11:194-205.

Zandenbergen, F., Madard, S., Escher, P., Tan, N. S., Patsouris, D., Jatkoe, T., Rojas-Caro, S., Madore, S., Wahli, W., Tafuri, S., Müller, M. and Kersten, S. 2005. The G0/G1 switch gene 2 is a novel PPAR target gene. Biochem. 392:313-24.

Zechner, R., Strauss, J. G., Haemmerle, G., Lass, A. and Zimmermann, R. 2005. Lipolysis: pathway under construction. Curr. Opin. Lipidol. 16:333-340.

Zhang, Y., Proenca, R., Maffei, M., Barone, M., Leopold, L. and Friedman, J. M. 1994. Positional cloning of the mouse obese gene and its human homologue. Nature 372:425-432.

Zhao, S. M., Ren, L. J., Chen, L., Zhang, X., Cheng, M. L., Li, W. Z., Zhang, Y. Y. and Gao, S. Z. 2009. Differential expression of lipid metabolism related genes in porcine muscle tissue leading to different intramuscular fat deposition. Lipids 44:1029-1037.

Zimmermann, R., Strauss, J. G., Haemmerle, G., Schoiswohl, G., Birner-Gruenberger, R., Riederer, M., Lass, A., Neuberger, G., Eisenhaber, F., Hermetter, A. and Zechner, R. 2004. Fat mobilization in adipose tissue is promoted by adipose triglyceride lipase. Science 306:13831386.

(Received Apr. 4, 2013; Revised May 15, 2013; Accepted Aug. 1, 2013) 